(C) Paul J. Lewi, 2005, 2006

Version of February 17, 2006

\title{
Speaking of Graphics
}

\section{Preface}

\section{On Graphicacy}

Graphics can be defined as translations of numbers in the form of a drawing, design or plan to explain or illustrate something. For our purpose, however, this definition is at the same time too broad and too narrow. In this essay we only consider graphics that are derived from tabulated observations or measurements. On the one hand, we omit technical, organizational and architectural drawings and graphics representing mathematical functions. On the other hand, we also extend the scope of graphics from explanation and illustration of known facts and relationships to the exploration and search for relevant questions and working hypotheses.

\section{Graphicacy as a tool of thought}

The quality of a graphic is usually related to its technical, perceptual or aesthetic properties, and to its information density. Here we also insist on 
the quality of a graphic as a mental model or projection of some relevant part of the world that we experience and as it is reflected in observations and measurements. In this sense, graphics are considered as objects of thought and imagination, as well as instruments of the mind. In our opinion, they can be likened to literary works, works of art, fashion designs, chemical formulas, musical scores, mathematical algorithms, computer programs and much more. The use and perfection of graphics forms part of our cultural and social heritage, as well as of technological and scientific progress. They are the product of a collective effort which constantly adds to an ever increasing repertoire, with distinctive genres and styles. In this essay, we describe the evolution from univariate graphics (bar charts and circular charts) via bivariate graphics (geographical maps, point and line charts) to the multivariate ones, especially the biplots, which describe relationships between objects (or subjects) and the multiple measurements (or observations) made on them. Some attention will be devoted also to the historical context and circumstances which have led to the development of particular types of graphic.

\section{The challenge of multivariate graphics}

Graphics speak languages of their own, which make them distinct from other modes of mental activity. Perhaps, they also appeal to specialized functions of the human brain. In order to understand graphical languages and to use them effectively, some graphical fluency (or graphicacy) is required. This applies especially to the multivariate types of graphics. 
One day multivariate graphics will be as commonplace as uni- and bivariate graphics are today. The computer hardware required to produce multivariate graphics is already available and the appropriate software is appearing steadily. A major obstacle, however, is that these multivariate graphics are still found difficult to read by the broader public, for lack of exposition of the particular language. For this purpose we present a kind of "grammar" for the language of basic multivariate graphics, especially the biplots. The latter are produced by the established methods of Principal Components Analysis and Correspondence Factor Analysis as well as by a closely related method which we have called Spectral Map Analysis. The latter forms a bridge between the former two methods. One may be deterred by the unfamiliarity and novelty of the vocabulary and syntactic rules that must be mastered in order to understand the new graphics. This truly constitutes a barrier that must be overcome. Some skeptics will maintain that the multivariate barrier is too high for those who are less educated in statistics and mathematics. Similar arguments, however, have been voiced before in earlier stages of the evolution of graphicacy. The barrier between bivariate graphics and multivariate graphics today is not substantially larger than the one between univariate graphics and bivariate diagrams in earlier days. For this reason we must pay little attention to the skeptics. Furthermore, after four centuries of emphasis on algebraic, logical and abstract formalisms, the pendulum seems to swing back again in the direction of geometric, artistic and poetic descriptions of nature and society.

\section{A pledge for graphicacy}


The past decades have been different from previous times by the rate at which quantitative data is generated and disseminated. On the one hand, the development of automatic equipment for the measurement of physical, chemical and biological phenomena has contributed to the increased generation and accumulation of quantitative data. At the same time, our means for conducting surveys, censuses and audits among the general population and among specific groups and organizations have multiplied and improved. On the other hand, the capacity of electronic devices for the storage, processing and sharing of this data doubles about every decade. The increased data flow must be attributed also to the widespread use of personal computers and workstations and to the popularization of the internet. Relational data bases organize the data such that they can be extracted and tabulated in every possible way. Spreadsheet programs are capable to dynamically transform such tables into other tables. By means of client-server systems one may combine tables from different sources and produce yet other tables. Parallel to the increase of our means for the generation, storage and dissemination of tabulated data, we also witness a revolution in the field of graphic display devices. These include color displays, high-resolution graphic printers and projection equipment. Yet, these advances in graphic computer hardware have not been paralleled by comparable developments in graphic methodology.

The average level of sophistication of graphics produced by contemporary graphic software is hardly better than that of the pre-computer era. The graphics offered today by popular graphics programs consist mainly of bar and pie charts and of point and line graphics in two dimensions or in threedimensional perspective viewing. Except for speed, colorfulness and 
technical quality, the personal computer and individual workstation have barely affected the quality of graphics in a fundamental way. Graphic technology is still far from exploiting its full capability for producing mind stirring graphics that would stimulate our inquisitiveness and imagination. On the contrary, some of the finer points of graphicacy are becoming outof-fashion because the services of professional draftsmen are less often called upon. This applies, for example, to logarithmic and probability charts, the use of which has been less well introduced to the general public. It points to a growing gap between the actual and potential state of graphical fluency. It calls for an effort to explain graphic languages to as broad a public as possible. If only this essay constitutes a small step in the direction of greater acceptance of modern (multivariate) graphics and of better understanding of the legacy of their predecessors, the author would feel rewarded for his undertaking.

\section{Acknowledgements}

Luc Wouters is acknowledged for taking the initiative to publish this work on the Internet and for taking care of its implementation. Annemie Biermans is thanked for secretarial help with the manuscript.

The author is particularly indebted to the late Dr. Paul Janssen, for his continuous guidance and for his uncompromising effort to design and use graphics as an integral part of original research and effective management. The ideas of Dr. Paul Janssen are reflected in the development of Spectral Map Analysis, a graphical multivariate method, which originated in drug research for the analysis of biological activity spectra. Subsequently, the approach found applications in marketing for 
competitive positioning, and in management for the analysis by characteristic ratios.

Hopefully, some illustrations from real life in this essay will convince the reader that the multivariate barrier is worth to be climbed and overcome. History shows that new ideas often meet with resistance, until their practical advantage and benefit become apparent.

Paul J. Lewi

Turnhout (Belgium), February 2006. 